\title{
The blue straggler S 1082: A triple system in the old open cluster M 67*
}

\author{
M. van den Berg ${ }^{1}$, J. Orosz ${ }^{1}$, F. Verbunt ${ }^{1}$, and K. Stassun ${ }^{2}$ \\ 1 Astronomical Institute, Utrecht University, PO Box 80000, 3508 TA Utrecht, The Netherlands \\ 2 Department of Astronomy, University of Wisconsin-Madison, 475 N Charter St, Madison WI 53706, USA
}

Received 7 February 2001 / Accepted 9 March 2001

\begin{abstract}
We present a photometric and spectroscopic study of the blue straggler S 1082 in the open cluster M 67 . Our observations confirm the previously reported 1.07 day eclipse light curve and the absence of large radialvelocity variations of the narrow-lined star. However, we find two more spectral components which do vary on the 1.07 day period. We conclude that the system is triple. We fit the light and radial-velocity curves and find that the total mass of the inner binary is more than twice the turnoff mass and that the outer companion to the binary is a blue straggler on its own account. We briefly discuss formation scenarios for this multiple system.
\end{abstract}

Key words. binaries: eclipsing - binaries: spectroscopic - blue stragglers - stars: individual: S 1082 - open clusters and associations: individual: M 67

\section{Introduction}

Blue stragglers are stars that are bluer and more luminous than the main-sequence turnoff of the population to which they belong. Hence they appear to be either younger than the other stars or have main-sequence lifetimes that exceed those of stars of similar mass. Blue stragglers are easiest to distinguish in globular and open clusters but are also found among field halo stars. Formation scenarios that require anomalous evolution of a single star are considered less likely than explanations that involve the interaction of stars in a binary or in a multiple-star encounter. For example, a blue straggler may be formed when one star in a binary accretes a substantial amount of mass from its companion or when two stars merge either in a binary or in a collision. In the last case, blue stragglers carry information about the high-stellar density environments in which they are found. Reviews on blue stragglers are given by e.g. Stryker (1993) and Bailyn (1995).

The blue stragglers in M 67 have received frequent attention. Ten of them were monitored for radial-velocity variations for nearly twenty years; this revealed one shortperiod (4.2 day) eccentric binary and five circular and ec-

Send offprint requests to: M. van den Berg,

e-mail: m.c.vandenberg@astro.uu.nl

* Based on observations made with the William Herschel Telescope, the Isaac Newton Telescope and the Jacobus Kapteyn Telescope operated on the island of La Palma by the Isaac Newton Group in the Spanish Observatorio del Roque de los Muchachos of the Instituto de Astrofisica de Canarias, and the Dutch Telescope operated at La Silla by the European Southern Observatory. centric binaries with periods between 850 and 5000 days (Milone \& Latham 1992; Latham \& Milone 1996). Leonard (1996) and Hurley et al. (2001) concluded that not one of the proposed blue-straggler formation mechanisms alone can explain the properties of these binaries.

S 1082 is one of the remaining four blue stragglers without an orbital solution. Its spectrum shows the lines of an early F-type subgiant. These lines show only small radial-velocity variations $\left(v_{\mathrm{rad}}<7 \mathrm{~km} \mathrm{~s}^{-1}\right.$ peak-to-peak, Mathieu et al. 1986) and little rotational broadening ( $v_{\text {rot }} \sin i=4-11 \mathrm{~km} \mathrm{~s}^{-1}$, van den Berg et al. 1999). This appears to be in contradiction to the eclipse light curve with a period of 1.07 days found by Goranskij et al. (1992). The relative velocity $v$ of two stars in a 1.07 day binary is $v=208\left(M / M_{\odot}\right)^{1 / 3} \mathrm{~km} \mathrm{~s}^{-1}$ where $M$ is the total mass of the binary; the stellar rotation in such a short-period binary is expected to be synchronised with the orbit, giving $v_{\text {rot }}=(R / a) v$, with $a$ the distance between the binary stars and $R$ the stellar radius. The eclipse light curve excludes the low inclination as well as the extreme mass ratio required to bring the observed velocity limits in agreement with the predicted values for an F star in the binary. We therefore tentatively conclude that S1082 is a (visual or physical) triple.

Evidence for a short orbital period is also furnished by the variation detected in a broad, shallow component (Mathys 1991) in e.g. the $\mathrm{H} \alpha$ line on time scales of hours (van den Berg et al. 1999). Evidence for a wide orbital period of about 1000 days was found from the radial-velocity variations of the narrow lines of the F star (Milone 1991). 
Table 1. Log of the photometric observations. For each run we give the date of the observations, the telescope and filters used and the typical exposure time for each filter.

\begin{tabular}{lllll}
\hline Run & Dates & Telescope & Filters & $t_{\text {exp }}(\mathrm{s})$ \\
\hline 1 & Feb. 8-19 1999 & 0.91m ESO & $U B$ & 300120 \\
& & Dutch & $V$ Gunn $i$ & 120120 \\
2 & Dec. 25, 26 1999 & 1m ING JKT & $B V$ & 7530 \\
3 & Feb. 13-16, 20 2000 & 1m ING JKT & $U$ U $V I$ & 35030 \\
& & & & 158 \\
\hline
\end{tabular}

We have collected multiband photometry of S 1082 together with high-resolution spectra that sample the light curves at different phases. Our goal was to investigate the eclipses found by Goranskij et al., to monitor the variability of the second spectral component as function of photometric phase and to model the light curve together with the radial-velocity curves. Section 2 describes the observations and data reduction. The results of the spectroscopic and light curve analysis are presented in Sects. 3 and 4. In Sect. 5 we summarise our interpretation of the nature of this blue straggler.

\section{Observations and data reduction}

\subsection{Photometry}

S 1082 was monitored in the $U, B, V, I$ and Gunn $i$ bands on three occasions (see Table 1 ). In run 1 we observed the star during twelve nights with the $0.91 \mathrm{~m}$ ESO-Dutch telescope at La Silla. The observing schedule was divided in four blocks of three nights; every first night the star was observed for an average of 5 consecutive hours in a $U B V$ Gunn $i$-exposure sequence while every second and third night typically one to three exposures were taken in each filter. During both nights of run 2, S1082 was observed for several hours with the $1 \mathrm{~m}$ Jacobus Kapteyn Telescope on La Palma. In run 3, also on the Jacobus Kapteyn Telescope, we aimed to complete the phase coverage of the light curve between phases 0-0.3.

Standard reduction steps of bias subtraction and flatfielding were performed with $\mathrm{IRAF}^{1}$ routines. Aperture photometry for all the stars in the field was done with the DAOPнот.PHOT task. Differential light curves for each individual dataset were computed with ensemble photometry (Honeycutt 1992). The variability properties of the other stars in the fields are discussed in van den Berg et al. (2001) and Stassun et al. (2001, in preparation). We refer the readers to these papers for a full description of the observations and the photometry reduction.

\footnotetext{
${ }^{1}$ IRAF is distributed by the National Optical Astronomy Observatories, which are operated by the Association of Universities for Research in Astronomy, Inc., under cooperative agreement with the National Science Foundation.
}

Table 2. Log of the spectroscopic observations. For each run we give the date of the observations, the wavelength coverage in $\AA$, the number of observations, the exposure time in seconds, the spectrograph used and (if applicable) the radial-velocity standard.

\begin{tabular}{|c|c|c|c|c|c|c|}
\hline Run & Dates & $\Delta \lambda$ & $\#$ & $t_{\exp }$ & Inst & RV \\
\hline \multirow[t]{3}{*}{1} & Feb. 281996 & $3920-4920$ & 2 & 600 & ues & HD $136202^{a}$ \\
\hline & & & & 300 & & \\
\hline & & 4890-7940 & 3 & 360 & ues & HD 136202 \\
\hline 2 & Feb. 162000 & $4380-8650$ & 17 & 1200 & ues & HD $89449^{b}$ \\
\hline 3 & Feb. 202000 & $3535-5035$ & 1 & 900 & ids & - \\
\hline 4 & Feb. 222000 & $3535-5035$ & 1 & 900 & ids & - \\
\hline 5 & Mar. 132000 & $4380-8650$ & 4 & 1800 & ues & HD 89449 \\
\hline 6 & Mar. 202000 & $4380-8650$ & 5 & 1200 & ues & HD 89449 \\
\hline
\end{tabular}

${ }^{a}$ F8III-IV ${ }^{b}$ F6IV (Simbad).

\subsection{Spectroscopy}

\subsubsection{High-resolution spectra}

High-resolution $(R \approx 49000)$ echelle spectra were taken with the Utrecht Echelle Spectrograph on the $4.2 \mathrm{~m}$ William Herschel Telescope on La Palma. S 1082 was observed on four nights in 1996 and 2000 (see Table 2 for a log of all spectroscopic observations).

The 1996-spectra were centred on a blue $(4250 \AA)$ and red $(5930 \AA)$ wavelength. The 31 lines $\mathrm{mm}^{-1}$ grating was used in combination with the $1024 \times 1024$ pixels $^{2}$ TEKCCD. For a full description of the spectra of run 1 we refer to van den Berg et al. (1999).

The spectra of 2000 were all taken with the same instrumental setup: the 79 lines $\mathrm{mm}^{-1}$ grating was used with the $2148 \times 2148$ pixels $^{2}$ SITe1-CCD while the spectra were centred on $5584 \AA$. In run 2 the seeing was about $2^{\prime \prime}$, while light clouds were present during the start of the run. The slit width was set to $1^{\prime \prime}$. To secure stability, no changes were made to the instrumental setup during the night. In the first two observations of run 5 the seeing was about $2^{\prime \prime}$; this deteriorated to $3^{\prime \prime}$ with cloudiness during the last two observations. As the slit width was kept fixed at $1^{\prime \prime}$, these spectra are of bad quality. In run 6 the seeing was $2^{\prime \prime}$ during the first two observations but later improved to 1.!6. The slit width was accordingly changed from 2 to 1 .' 2 . Due to the wider slit these spectra have a lower resolution than the spectra of run 1 and 5. All frames were exposed for $1200 \mathrm{~s}$, except for those of run 5 that were exposed for $1800 \mathrm{~s}$ to account for the bad seeing conditions. During each run we observed radial-velocity standards. Flatfield images were made with exposures of a tungsten lamp. Thorium-argon lamp emission-line spectra were taken for wavelength calibration. Data-reduction was done in IRAF with CCDRED and ECHELLE routines. After correcting the frames for the electronic bias and after flat fielding, spectra for each 
echelle-order were extracted with optimal extraction. Towards the red, gaps occur in the wavelength coverage. For the wavelength calibration, fifth-order polynomials were fitted in both directions to the positions of the arclines on the CCD; the maximum residuals to the fit were $\sim 0.0125 \AA$ corresponding to $0.75 \mathrm{~km} \mathrm{~s}^{-1}$. Low-order polynomials were fitted to the spectra for continuum normalisation.

\subsubsection{Intermediate-resolution spectra}

Two intermediate-resolution $(R \approx 3600)$ spectra were obtained with the Intermediate Dispersion Spectrograph (IDS) mounted on the $2.5 \mathrm{~m}$ Isaac Newton Telescope on La Palma, on February 20.90214, 2000 (UT) and February 22.85523 (UT). The R1200B grating and the EEV10 CCD combination gave a spectral resolution of $1.19 \AA$ $(F W H M)$ and a useful wavelength range of 3533-4825. The exposure times were $900 \mathrm{~s}$ each, and the signal-tonoise level in the extracted spectra ranged from about 65 per pixel near the Balmer jump to about 150 near $4750 \AA$.

\section{Results}

\subsection{Eclipse light curve}

The new light curves of S1082 confirm the findings of Goranskij et al. (1992) and fill in the gap in their data between photometric phase 0.53 and 0.74 (with phase 0 corresponding to primary minimum). In Fig. 1 our data are folded on their ephemeris for the primary minimum:

MinI $=2444643.253(5)+1$ d.0677978(50) E.

Two unequal eclipses with an amplitude of about 0.09 and $0.05 \mathrm{mag}$ in $V$ are clearly visible with the deeper, primary eclipses occuring around phase 0 as expected from Eq. (1). Goranskij et al. note that the light curve near second quadrature (phase 0.75 ) is systematically higher than near first quadrature (phase 0.25 ) by 0.01 to $0.02 \mathrm{mag}$ in $V$. This might also be the case in our data, but the scatter between phases 0.1 and 0.3 makes this difficult to see. The scatter is probably related to the bad observing conditions (nearby moon) of the last run.

\subsection{Spectral line profiles}

The spectra of S1082 are dominated by a narrow-lined component resembling a star of type early-F. In addition, the presence of a broad and shallow component in the spectra is prominent in the $\mathrm{H} \alpha$ line (see also van den Berg et al. 1999). When the spectra are arranged in order of light curve phase using Eq. (1), it is clear that the position and strength of the asymmetric absorption in the wings vary regularly (Fig. 2); the spectra that were included in Fig. 8 of van den Berg et al. (1999) correspond to photometric phases $0.092,0.16$ and 0.25 . Around first quadrature (phase 0.25) the depth of $\mathrm{H} \alpha$ is smallest while

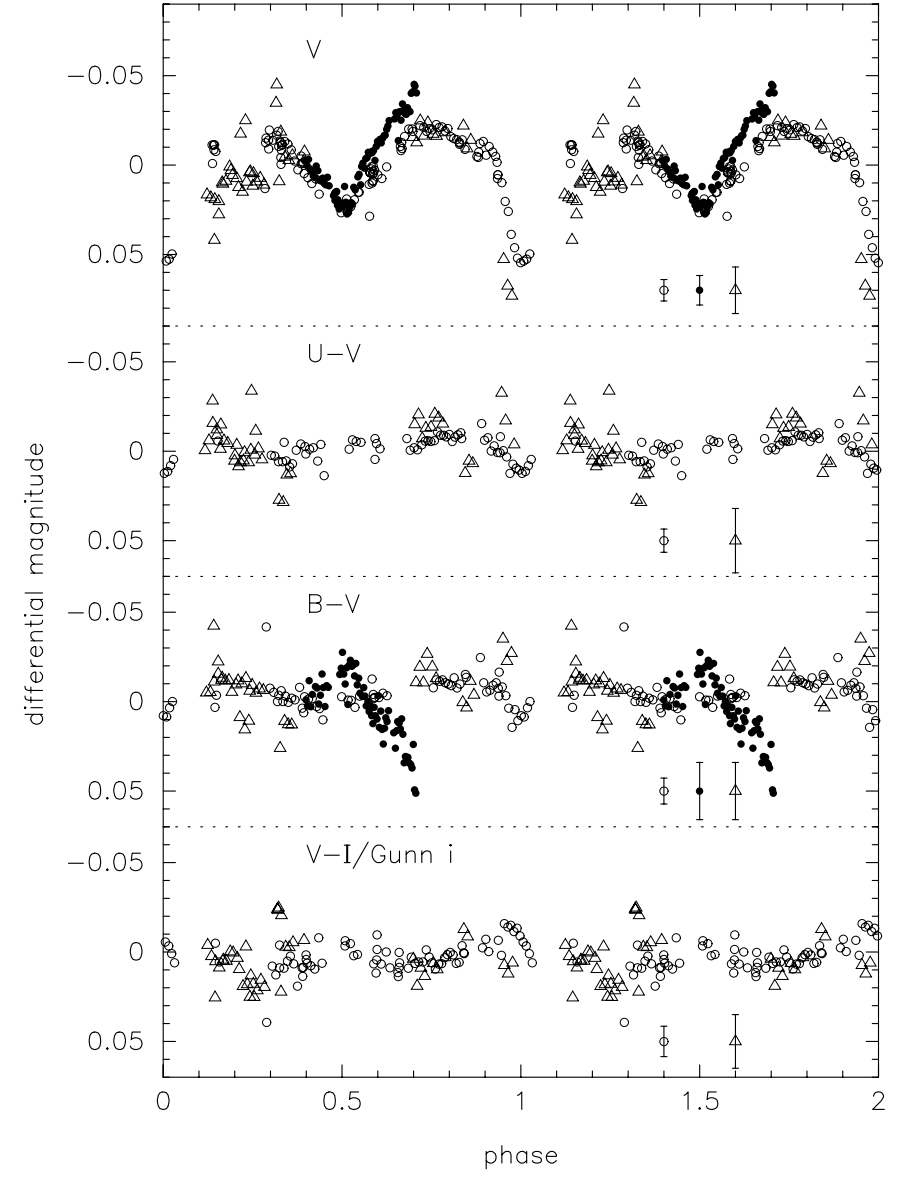

Fig. 1. Light and colour curves for S1082 folded on the ephemeris of Goranskij et al. (1992). Data from the three runs are indicated with different symbols: open circles for run 1, filled circles for run 2 and triangles for run 3. A typical error bar is shown for each run. Magnitude and colours are plotted relative to the average $V(11.25), U-V(0.45), B-V(0.41)$ and $V-I(0.57)$ values in our measurements.

the asymmetric absorption in the wings is maximally blueshifted; for phases between 0.5 and 1 the phase-coverage is sparser, but in the spectra of phases 0.88 and 0.90 it can be seen that the asymmetric absorption has moved to the red wing. The timing of the velocity shift with respect to the eclipses associates the broad-lined feature with the brighter star in the eclipsing binary (the brighter star must approach us after the primary eclipse). Similar variable line profiles are visible in the $\mathrm{H} \beta$, $\mathrm{Na}$ I D, Mg I b and CaII infrared lines (Fig. 3).

The temperature of the narrow-lined star in S1082 can be measured with the low-resolution spectra. We assume that its light is least contaminated by its companion star(s) in the blue and therefore concentrate on the region around the Balmer jump. In Fig. 4 synthetic spectra for various effective temperatures $T_{\text {eff }}$ are compared with the IDS spectrum between $3535 \AA$ and $4050 \AA$. The spectra were computed with model atmospheres for solar metallicity (Kurucz 1979). The observed spectrum was corrected for the reddening towards M $67(E(B-V)=$ $0.032 \pm 0.006$, Nissen et al. 1987) and for a radial-velocity 


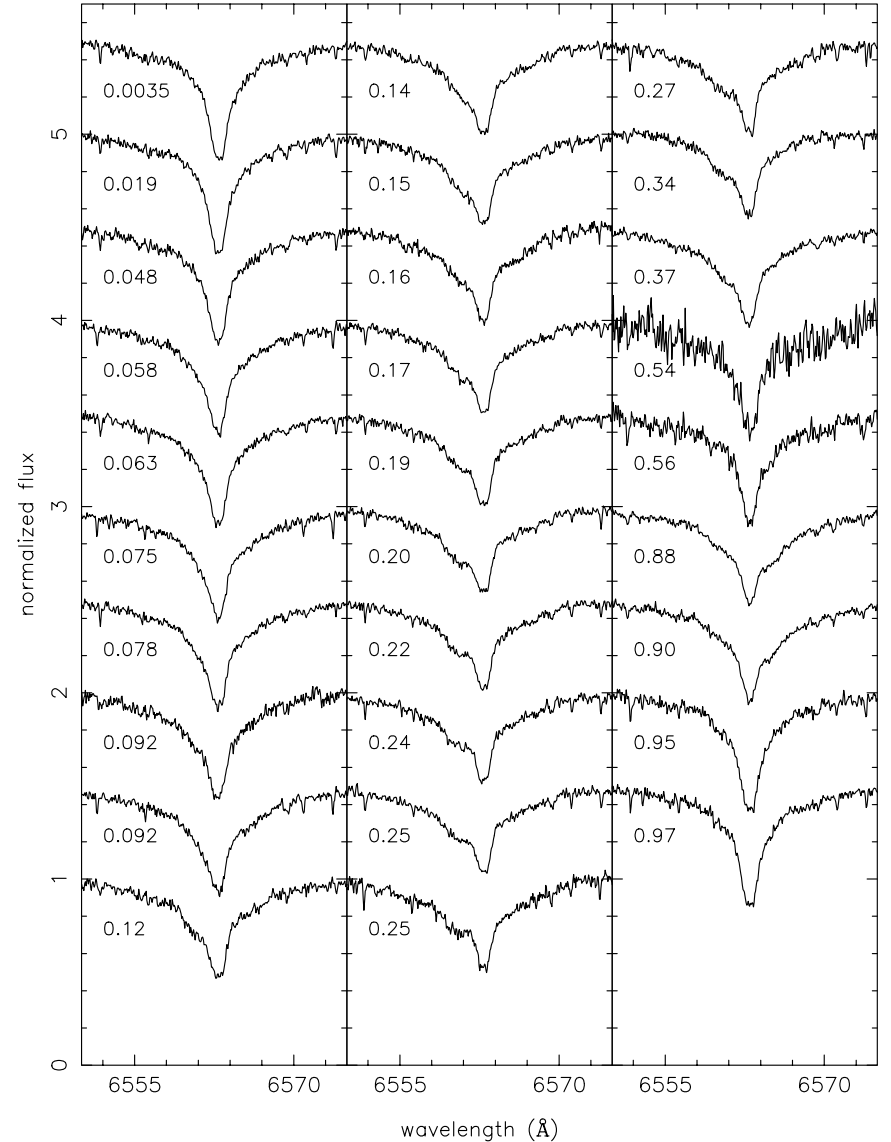

Fig. 2. Series of $\mathrm{H} \alpha$ profiles in $\mathrm{S} 1082$. The phase of the light curve is indicated for each spectrum. Note the central depth of the line and the asymmetric wings. The observations during phase 0.54 and 0.58 are noisy due to the bad weather conditions of run 5. The continuum separation between the spectra is 0.5 unit.

difference with respect to the model spectra. From the relative strength of the CaII H\&K lines, a sensitive temperature indicator in this region (e.g. Gray \& Garrison 1989), and the contrast in the Balmer jump it is clear that the observed spectrum is hotter than that of a $6500 \mathrm{~K}$ star.

This part of the spectrum was fitted to model spectra with surface gravity $\log g$ ranging from 0.5 to 5.0 in steps of $0.5, T_{\text {eff }}$ from 6000 to $8000 \mathrm{~K}$ in steps of $250 \mathrm{~K}$ and a fixed projected rotational velocity $v_{\text {rot }} \sin i$ of $10 \mathrm{~km} \mathrm{~s}^{-1}$. A free parameter is a wavelength-independent scale factor ranging from 0.025 to 1 in increments of 0.025 that is a measure of the relative contribution of the narrow-lined star. Observed and model spectra were normalised to the flux at $4050 \AA$. A straight line was fitted to the difference between the observed and each scaled model spectrum. The model that produces the smallest residuals to the fit has $T_{\text {eff }}=7500 \mathrm{~K}, \log g=4.5$ and scale factor 0.85 . If the temperature of the hot star is indeed $7500 \mathrm{~K}$, it is a late A rather than an F star; the value of $\log g$ is close to that of a main-sequence star for which $\log g \approx 4.25$ (Gray 1992). This shows that the narrow-lined component dominates the spectrum in the blue and it implies that one of the in-

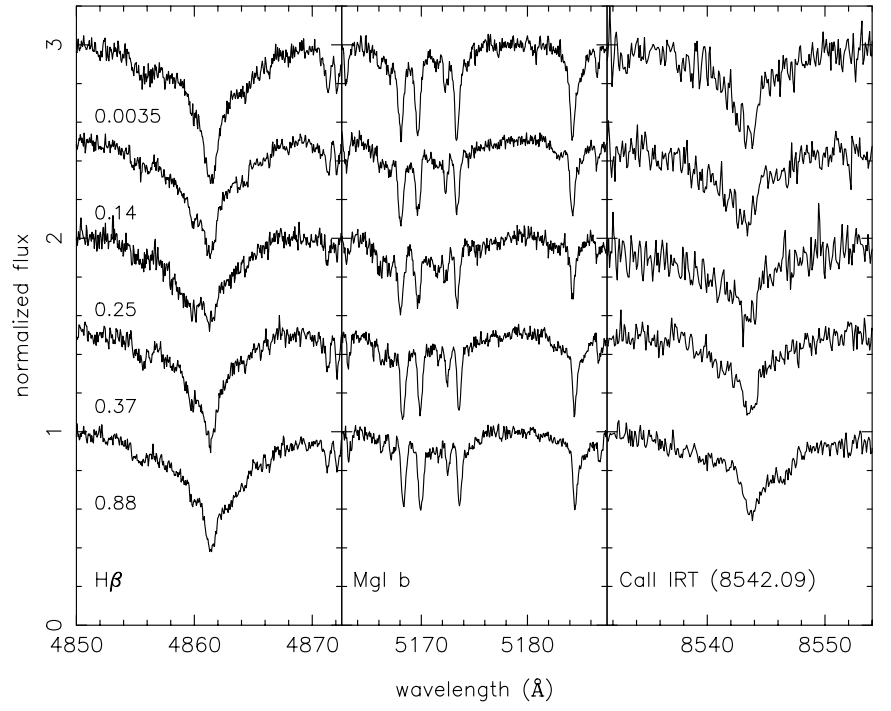

Fig. 3. $\mathrm{H} \beta, \mathrm{Mg}$ Ib and Ca II IRT lines in S 1082 for a selected set of observations. Light curve phases are indicated. The continuum separation between the spectra is 0.5 unit.

dividual components of S 1082 remains a blue straggler. A more accurate decomposition of S 1082 is given in Sect. 4 .

\subsection{Radial-velocity curves}

Radial velocities were measured via cross correlation of the high-resolution spectra with appropriate template spectra (Table 3). To determine the radial velocities of the narrow-lined star in S 1082 we adopted as templates spectra of F-type radial-velocity standards observed during the same run (Table 2). Velocities were derived for each order separately. Only orders without strong telluric lines were selected, and included 4890-6820 $\AA$ for the 1996-spectra and 4435-6820 $\AA$ for the 2000-spectra. The radial velocities listed in the third column of Table 3 are the averages of the values from the individual orders; the errors represent the spread around the average. Note that systematic errors can still be included, e.g. due to the wavelength calibration. We expect the latter not to exceed $0.75 \mathrm{~km} \mathrm{~s}^{-1}$ (see Sect. 2.2.1). Our measurements confirm that the narrowlined component in S 1082 shows radial-velocity variations of only a few $\mathrm{km} \mathrm{s}^{-1}$.

The radial-velocity curves of the stars in the eclipsing binary were measured after the contribution of the hot, narrow-lined star to the total spectrum was removed. To that end, all spectra were first brought to the rest frame of the hot star. Then, a hot-star template spectrum was constructed by taking the median of the spectra obtained at phases $0.95,0.97,0.0035,0.019$ and 0.048; these particular phases were chosen in order to let the spectral line profiles of the template be as symmetric as possible. For the 1996-spectra, taken with a different instrumental setup, the spectrum obtained at phase 0.092 was chosen as a template. The relative contribution of the hot-star template to the remaining spectra was derived with the same fitting procedure as described in Sect. 3.2: the template 


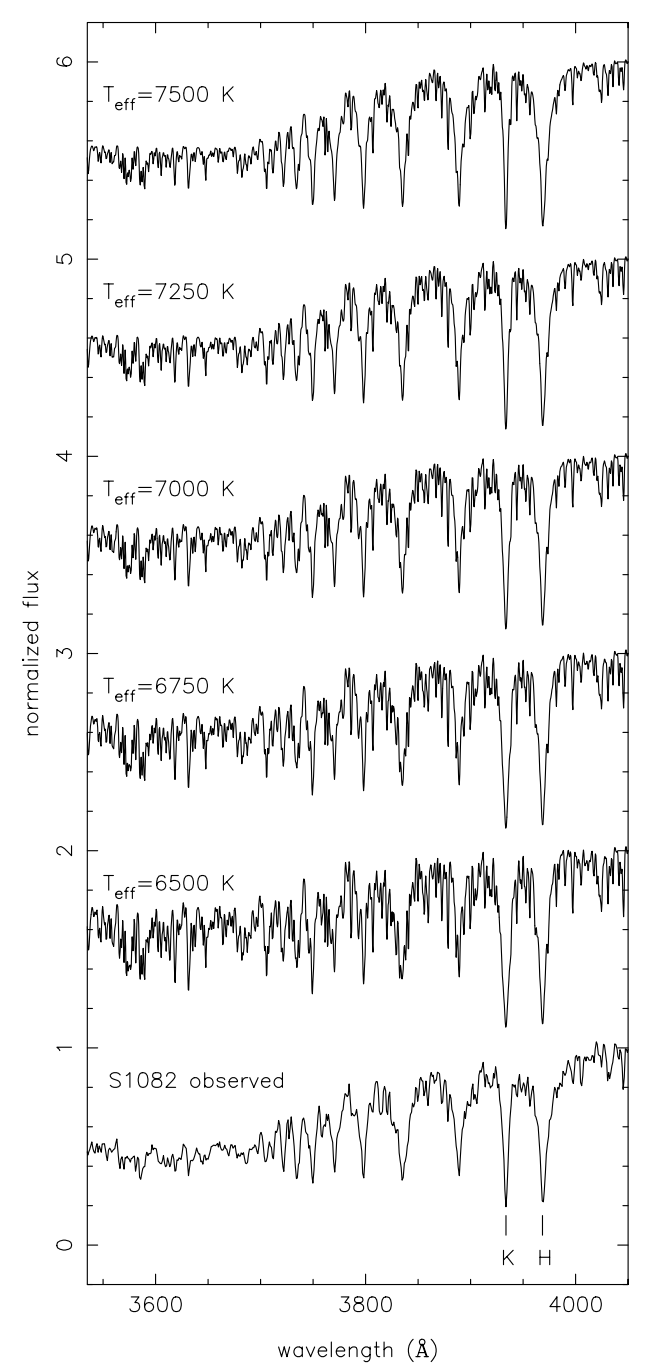

Fig. 4. Spectrum of S 1082 compared with synthetic spectra of various effective temperatures; surface gravity and projected rotational velocity are fixed at $\log g=4.5$ and $v_{\text {rot }} \sin i=$ $10 \mathrm{~km} \mathrm{~s}^{-1}$. The spectra are normalised to the flux at $4050 \AA$; each spectrum is offset with 1 unit from the previous spectrum. The relative strength of the $\mathrm{Ca}$ II $\mathrm{K}$ line and the $\mathrm{Ca}$ II $\mathrm{H}+\mathrm{H} \epsilon$ blend (marked with "K" and "H"), and the contrast in the Balmer jump (flux near $4050 \AA$ relative to flux near $3600 \AA$ ) are indicators of temperature.

multiplied with scaling factors ranging from 0.025 to 1 was subtracted from each individual spectrum, and the scaling that produces the smallest residuals around a fit to a straight line was chosen as the appropriate scaling for that particular spectrum. The scaled template was then subtracted from the total spectrum to obtain the spectrum of the binary at various phases.

Next, these residual spectra were correlated against a synthetic spectrum of $T_{\text {eff }}=5250 \mathrm{~K}$ and $\log g=3.5$. For this model spectrum we chose $v_{\text {rot }} \sin i=50 \mathrm{~km} \mathrm{~s}^{-1}$ to roughly match the apparently broader lines in the spectrum of the binary. This procedure was repeated for every order between 4380 and $6435 \AA$, excluding the order that contains the broad $\mathrm{H} \beta$ line and the region between 5690 and $6090 \AA$ for which the cross correlation functions

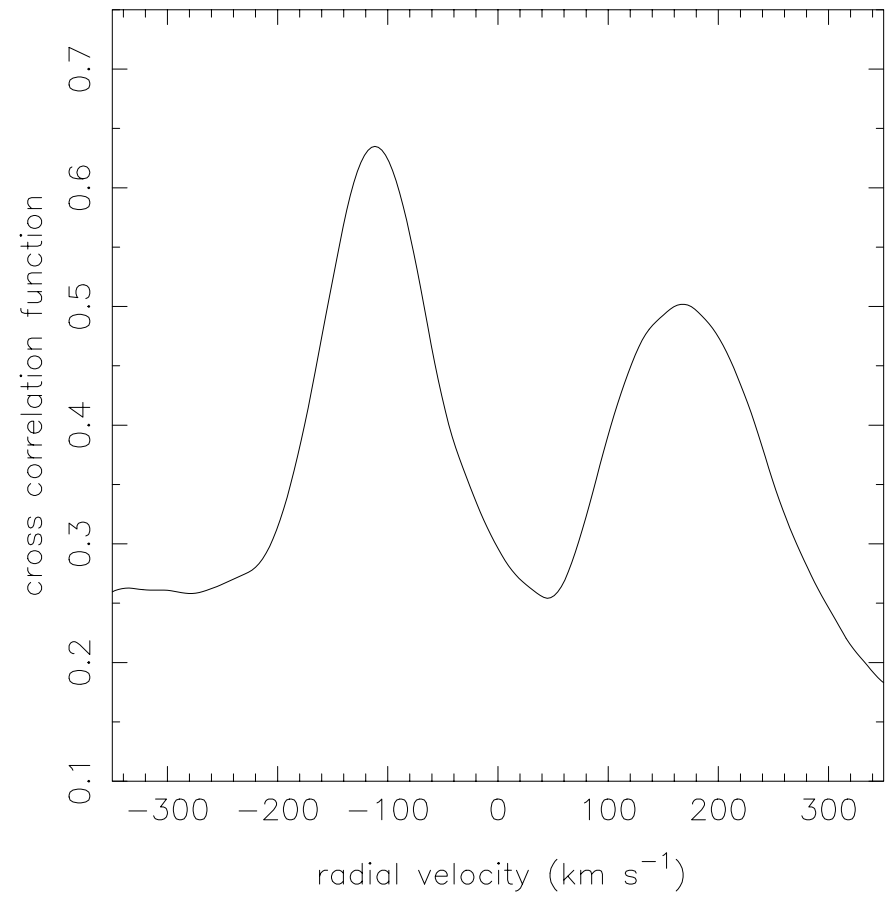

Fig. 5. Cross correlation function resulting from the residual spectrum taken at photometric phase 0.2535 after the contribution of the hot star is removed. The left peak corresponds to the primary star Aa in the eclipsing binary. The width of peak corresponding to the secondary $\mathrm{Ab}$ is broader which indicates that its spectral lines are more broadened by rotation. For this particular cross correlation the spectrum between 5160 and $5240 \AA$ was used. The radial velocity is given with respect to the hot star B in S 1082 .

are very noisy with no clear peaks. A cross correlation peak is sometimes visible at $v_{\text {rad }}=0 \mathrm{~km} \mathrm{~s}^{-1}$ and represents features of the hot star that were not corrected for (or introduced into the spectrum) by subtraction of the template. The cross correlation functions of most spectra clearly show two peaks with a variable separation (see Fig. 5). One peak is smaller and broader than the other, and is redshifted with respect to the template for phases smaller than 0.5 ; therefore, this peak is identified with the secondary star in the eclipsing binary. This is the first spectroscopic evidence for the third star in S1082. The measurements of the velocity of the primary near phase 0.1 are probably distorted due to its eclipse by the secondary star. In the following we will refer to the components of the eclipsing binary as $\mathrm{Aa}$ and $\mathrm{Ab}$, and to the outer companion as B.

We used the spectrum observed near first quadrature (wavelength region 5160 to $5240 \AA$ containing the $\mathrm{Mg} \mathrm{I} \mathrm{b}$ triplet) to derive an estimate for the projected rotational velocities of both components from the widths of their cross correlation peaks. To that end, we cross correlated a synthetic spectrum of a certain temperature $(\log g=4.0$, $v_{\text {rot }} \sin i=30 \mathrm{kms}^{-1}$ ) with synthetic templates of the same temperature and surface gravity with $v_{\text {rot }} \sin i$ ranging from 10 to $100 \mathrm{~km} \mathrm{~s}^{-1}$, and with the object spectrum. The peak of the cross correlation functions were 
Table 3. Radial velocities of the three stars in S1082, the components of the eclipsing binary $\mathrm{Aa}$ and $\mathrm{Ab}$, and the outer companion B. From left to right: heliocentric Julian date $(-2450000)$ at the midpoint of observation; photometric phase computed with the ephemeris of Goranskij et al. (1992); heliocentric radial velocity for star B; radial velocity of the primary $\mathrm{Aa}$ and secondary $\mathrm{Ab}$ in the eclipsing binary with respect to star B in $\mathrm{km} \mathrm{s}^{-1}$. The spectra of phases 0.5378 and 0.5609 were taken under bad observing conditions.

\begin{tabular}{|c|c|c|c|c|}
\hline $\begin{array}{r}\text { HJD- } \\
2450000\end{array}$ & $\begin{array}{l}\text { phot. } \\
\text { phase }\end{array}$ & $\begin{array}{l}v_{\mathrm{rad}, \mathrm{B}} \\
\mathrm{km} \mathrm{s}^{-1}\end{array}$ & $\begin{array}{c}v_{\mathrm{rad}, \mathrm{Aa}} \\
\mathrm{km} \mathrm{s}^{-1}\end{array}$ & $\begin{array}{c}v_{\mathrm{rad}, \mathrm{Ab}} \\
\mathrm{km} \mathrm{s}^{-1}\end{array}$ \\
\hline 142.5101 & .09213 & $33.0 \pm 0.1$ & - & - \\
\hline 142.5790 & .1567 & $33.2 \pm 0.1$ & $-97 \pm 4$ & - \\
\hline 142.6837 & .2548 & $32.9 \pm 0.2$ & $-108 \pm 3$ & - \\
\hline 1591.3642 & .9540 & $33.5 \pm 0.4$ & - & - \\
\hline 1591.3822 & .9709 & $33.7 \pm 0.3$ & - & - \\
\hline 1591.4170 & .003451 & $34.2 \pm 0.3$ & - & - \\
\hline 1591.4334 & .01880 & $33.7 \pm 0.3$ & - & - \\
\hline 1591.4640 & .04750 & $32.4 \pm 0.3$ & - & - \\
\hline 1591.4805 & .06294 & $32.8 \pm 0.3$ & - & - \\
\hline 1591.4967 & .07812 & $32.7 \pm 0.3$ & $-73 \pm 4$ & $122 \pm 7$ \\
\hline 1591.5452 & .1235 & $33.2 \pm 0.4$ & $-96 \pm 3$ & $148 \pm 4$ \\
\hline 1591.5616 & .1389 & $33.3 \pm 0.4$ & $-95 \pm 2$ & $141 \pm 4$ \\
\hline 1591.5779 & .1541 & $33.3 \pm 0.4$ & $-100 \pm 2$ & $153 \pm 5$ \\
\hline 1591.5993 & .1742 & $33.1 \pm 0.5$ & $-104 \pm 2$ & $154 \pm 5$ \\
\hline 1591.6150 & .1890 & $33.5 \pm 0.4$ & $-108 \pm 2$ & $150 \pm 12$ \\
\hline 1591.6310 & .2039 & $33.9 \pm 0.5$ & $-107 \pm 3$ & $166 \pm 5$ \\
\hline 1591.6474 & .2192 & $34.2 \pm 0.5$ & $-109 \pm 2$ & $170 \pm 8$ \\
\hline 1591.6682 & .2387 & $33.8 \pm 0.5$ & $-113 \pm 2$ & $160 \pm 7$ \\
\hline 1591.6840 & .2535 & $33.9 \pm 0.5$ & $-113 \pm 3$ & $169 \pm 14$ \\
\hline 1591.7010 & .2695 & $33.9 \pm 0.5$ & $-115 \pm 2$ & $180 \pm 5$ \\
\hline 1617.4072 & .3435 & $31.8 \pm 0.4$ & $-104 \pm 2$ & $163 \pm 5$ \\
\hline 1617.4308 & .3656 & $32.7 \pm 0.5$ & $-96 \pm 2$ & $153 \pm 5$ \\
\hline 1617.6147 & .5378 & $36.0 \pm 1.2$ & $28 \pm 5$ & - \\
\hline 1617.6394 & .5609 & $35.2 \pm 0.8$ & - & - \\
\hline 1624.3903 & .8832 & $35.4 \pm 0.4$ & $91 \pm 3$ & $-130 \pm 5$ \\
\hline 1624.4092 & .9009 & $34.6 \pm 0.4$ & $85 \pm 4$ & $-132 \pm 7$ \\
\hline 1624.5766 & .05765 & $32.4 \pm 0.3$ & - & - \\
\hline 1624.5949 & .07484 & $32.7 \pm 0.3$ & $-80 \pm 4$ & $121 \pm 6$ \\
\hline 1624.6131 & .09188 & $32.6 \pm 0.4$ & $-90 \pm 4$ & $128 \pm 5$ \\
\hline
\end{tabular}

fitted with Gaussian profiles. This was repeated for synthetic spectra of temperatures 5000 to $6750 \mathrm{~K}$ in steps of $250 \mathrm{~K}$. With the results we constructed calibration curves for each temperature that give the width of the cross correlation peak as a function of the projected rotational velocity of the synthetic template. The widths of the cross correlation peaks from the object spectrum then give a rough estimate for the $v_{\text {rot }} \sin i$ of both stars. For star Aa we obtain a $v_{\text {rot }} \sin i$ of $51 \mathrm{~km} \mathrm{~s}^{-1}(5750 \mathrm{~K})$ to $58 \mathrm{~km} \mathrm{~s}^{-1}(6750 \mathrm{~K})$, for star Ab a $v_{\text {rot }} \sin i$ of $83 \mathrm{~km} \mathrm{~s}^{-1}$ $(5500,5750 \mathrm{~K})$ to $87 \mathrm{~km} \mathrm{~s}^{-1}(6750 \mathrm{~K})$.

\section{Parameter estimation for the eclipsing binary}

We used the recently written Eclipsing Light Curve (ELC) code of Orosz \& Hauschildt (2000) to fit the basic binary observables of S1082, namely the light curves in the four filters, the two velocity curves and the observed projected rotational velocities of the two stars $\mathrm{Aa}$ and $\mathrm{Ab}$. The blue straggler B in this case is modelled as "third light". The addition of a constant (in phase) light will reduce the observed relative amplitudes of the light curves. The ELC code uses model atmosphere specific intensities, so the flux from a third star can be self-consistently added to several different bandpasses (i.e. a blue third light star dilutes the $B$-band light curve of a red binary more than it dilutes the $I$-band light curve). We mainly used the specific intensities derived from the NEXTGEN models (Hauschildt et al. 1999a, 1999b). A few models were also computed using specific intensities derived from Kurucz (1979) models. Only the photometric data of run 1 were used for the light curve fitting as they form the largest set of homogeneous measurements.

We start by estimating the component masses of the eclipsing binary. Unfortunately, we only have radial velocities near one quadrature phase (poor weather prevented us from observing the opposite quadrature), so the velocity amplitudes of the two curves and the binary systemic velocity will not be as constrained as we would like them to be. We fit sinusoids to each of the velocity curves, fixing the period at the photometric period. For star Aa we find $K_{\mathrm{Aa}}=120.8 \pm 6.4 \mathrm{~km} \mathrm{~s}^{-1}, \gamma_{\mathrm{rel}, \mathrm{Aa}}=1.7 \pm 5.8 \mathrm{~km} \mathrm{~s}^{-1}$, $\phi_{0, \mathrm{Aa}}$ (spect) $=0.747 \pm 0.007$, where $\gamma_{\mathrm{rel}}$ is the systemic velocity relative to the mean cluster velocity and where $\phi_{0}$ (spect) refers to the phase of maximum velocity. For star $\mathrm{Ab}$ we find $K_{\mathrm{Ab}}=190.1 \pm 12.5 \mathrm{~km} \mathrm{~s}^{-1}, \gamma_{\mathrm{rel}, \mathrm{Ab}}=$ $-2.6 \pm 12.5 \mathrm{~km} \mathrm{~s}^{-1}, \phi_{0, \mathrm{Ab}}$ (spect) $=0.242 \pm 0.010$. The errors on the individual velocities were scaled to yield $\chi_{\nu}^{2}=1$ for each curve, and the error estimates on the fitted parameters were derived using the scaled uncertainties. The phasing of the curves are consistent with expectations, where star Aa has its maximum velocity one fourth of an orbital cycle before the deeper photometric eclipse. Since systemic velocities are consistent with being zero, we will assume the binary is a cluster member and hence has $\gamma_{\mathrm{rel}}=0 \mathrm{~km} \mathrm{~s}^{-1}$. In this case we find for star Aa $K_{\mathrm{Aa}}=$ $119.1 \pm 3.1 \mathrm{~km} \mathrm{~s}^{-1}$ and $\phi_{0, \mathrm{Aa}}$ (spect) $=0.748 \pm 0.007$, and for star $\mathrm{Ab}$ we find $K_{\mathrm{Ab}}=190.1 \pm 11.6 \mathrm{~km} \mathrm{~s}^{-1}$ and $\phi_{0, \mathrm{Ab}}$ (spect) $=0.243 \pm 0.010$. Taking the sinusoid fits at face value, we can immediately compute the mass ratio of the binary. We find $Q \equiv M_{\mathrm{Ab}} / M_{\mathrm{Aa}}=K_{\mathrm{Aa}} / K_{\mathrm{Ab}}=$ $0.63 \pm 0.04$. The minimum masses of the two component stars are then $M_{\mathrm{Aa}} \sin ^{3} i=2.01 \pm 0.38 M_{\odot}$ and $M_{\mathrm{Ab}} \sin ^{3} i=1.26 \pm 0.27 M_{\odot}$ (see Table 4 for a summary of the observed parameters of the eclipsing binary).

There are a total of twelve free parameters in the model: the filling factors (by radius) of the two stars $f_{\mathrm{Aa}}$, $f_{\mathrm{Ab}}$, the mean temperatures of the two stars $T_{\mathrm{Aa}}, T_{\mathrm{Ab}}$, the "spin factors" of the two stars $\Omega_{\mathrm{Aa}}, \Omega_{\mathrm{Ab}}$, where $\Omega$ is the ratio of the rotational angular velocity to the orbital angular velocity, the inclination $i$, the mass ratio $Q$, the orbital separation $a$, the temperature of the third light star $T_{\mathrm{B}}$, the surface gravity of the third light star $\log g_{\mathrm{B}}$, and the third light scaling factor $S A_{\mathrm{B}}$. We assume the orbit is circular, and that the third light star B does not vary. The gravity darkening exponent of star Aa was fixed at 0.25 , the standard value for a star with a radiative 


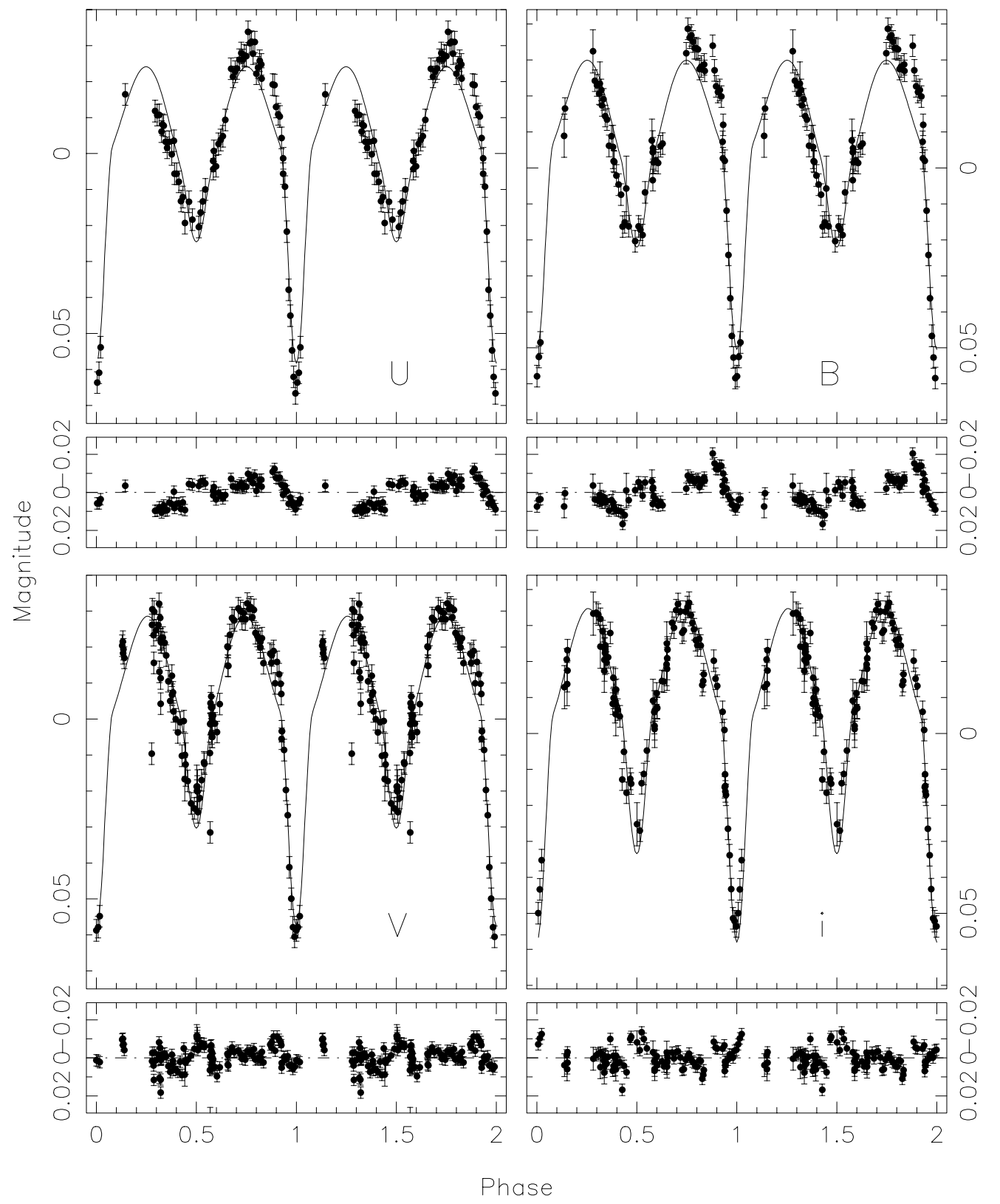

Fig. 6. Fit to the light curves in the $U, B, V$ and Gunn $i$ band. The small panels show the residuals to the fit. Only the data from the first observation run are used.

envelope, while the gravity darkening exponent for star $\mathrm{Ab}$ was set at the standard convective value of 0.08 . The ELC code uses Wilson's (1990) detailed reflection scheme, and for this problem the albedo of star Aa was taken to be 1 and the albedo of star Ab was taken to be 0.5. Three iterations of the reflection scheme were needed to achieve convergence. Both stars in the binary are assumed to be free of spots. A variation of the "grid search" routine outlined in Bevington (1969) was used to optimise the fits. In practice the fitting procedure involved a great deal of interaction where some parameters were temporarily fixed at certain values. Several two-dimensional grids in parameter space were defined (for example a grid of points in the $f_{\text {Aa }}, f_{\text {Ab }}$ plane). For each point, we fixed those parameters at the values defined by the grid location and optimised the other parameters, creating contours of $\chi^{2}$ values. New parameter sets were optimised using the set of parameters for a nearby point that gave the best previous fit. After the lowest $\chi^{2}$ value in a grid was found, then a new grid using other parameters was computed using the best solution as a starting point. The fitting took several weeks of CPU time on an Alpha XP 1000, and sampled a wide range of parameter space. We are reasonably confident that our results are at or very near the global $\chi^{2}$ minimum.

We found a relatively large number of solutions with similar $\chi^{2}$ values. Figure 6 shows a typical fit. The light curves in the 4 bands (Fig. 6) are fitted reasonably well, although there are still some systematic deviations, especially near the eclipse phases. The radial velocities are also fitted reasonably well (Fig. 7), but again there are some 
Table 4. Observed binary parameters. Aa and Ab refer to the stars in the eclipsing binary. $V$ and $B-V$ are taken from Montgomery et al. (1993).

\begin{tabular}{lr}
\hline parameter & value \\
\hline$P_{\text {phot }}$ & $1.0677971(7)$ \\
$T_{0}$ & $2444643.250(2)$ \\
$V$ & 11.251 \\
$B-V$ & 0.415 \\
$K_{\mathrm{Aa}}\left(\mathrm{km} \mathrm{s}^{-1}\right)$ & $119.1(3.1)$ \\
$K_{\mathrm{Ab}}\left(\mathrm{km} \mathrm{s}^{-1}\right)$ & $190.1(11.6)$ \\
$Q$ & $0.63(4)$ \\
$\gamma_{\mathrm{Aa}}\left(\mathrm{km} \mathrm{s}^{-1}\right)$ & $0.0[$ fixed] \\
$\gamma_{\mathrm{Ab}}\left(\mathrm{km} \mathrm{s}^{-1}\right)$ & $0.0[$ fixed] \\
$M_{\mathrm{Aa}} \sin ^{3} i\left(M_{\odot}\right)$ & $2.01(38)$ \\
$M_{\mathrm{Ab}} \sin ^{3} i\left(M_{\odot}\right)$ & $1.26(27)$ \\
$v_{\mathrm{rot}, \mathrm{Aa}} \sin i\left(\mathrm{~km} \mathrm{~s}^{-1}\right)$ & $56(5)$ \\
$v_{\mathrm{rot}, \mathrm{Ab}} \sin i\left(\mathrm{~km} \mathrm{~s}^{-1}\right)$ & $83(5)$ \\
\hline
\end{tabular}

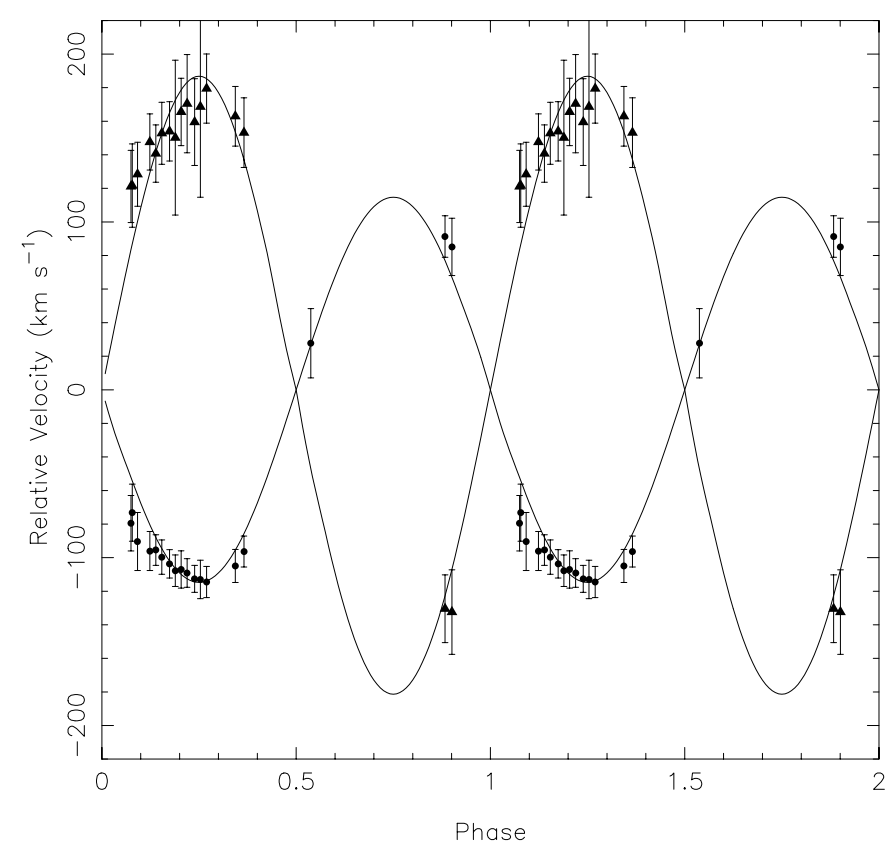

Fig. 7. Radial-velocity curves of star Aa of the eclipsing binary in S 1082 (filled circles) and star Ab (filled triangles). The lines indicate the best-fitting velocity curves as computed by ELC.

small systematic deviations (the velocity curves seem to be systematically flatter than the sinusoid fits near phase $0.25)$. In all solutions the binary is detached, i.e. both stars are well within their respective Roche lobes. We applied two additional constraints in order to narrow down the range of parameters. The first constraint is that the total $V$ magnitude and $B-V$ colour of the model should match the observed $V$ magnitude of $\mathrm{S} 1082$. In this case the apparent $V$ magnitude of the model is easy to compute. We used the synthetic photometry computed from the NextGen models ${ }^{2}$ to compute expected absolute $V$ mag-

\footnotetext{
2 ftp://calvin.physast.uga.edu/pub/NextGen/Colors/
}

Table 5. Fitted binary parameters. $\mathrm{Aa}$ and $\mathrm{Ab}$ refer to the stars in the eclipsing binary, B to the third, outer star.

\begin{tabular}{lrrr}
\hline parameter & $\mathrm{Aa}$ & $\mathrm{Ab}$ & $\mathrm{B}$ \\
\hline$f$ & $0.520(10)$ & $0.700(10)$ & \\
$R\left(R_{\odot}\right)$ & $2.07(7)$ & $2.17(3)$ & $\approx 2.5$ \\
$R / R_{\text {Roche }}$ & $0.66(7)$ & $0.86(7)$ & \\
$\Omega$ & $0.49(5)$ & $0.91(5)$ & \\
$T(\mathrm{~K})$ & $6480(25)$ & $5450(40)$ & $7500(50)$ \\
$\log g(\mathrm{cgs})$ & $4.21(2)$ & $4.0(2)$ & $4.25(5)$ \\
$M\left(M_{\odot}\right)$ & $2.70(38)$ & $1.70(27)$ & $\approx 1.7$ \\
$V$ & $12.33(11)$ & $13.10(11)$ & $12.24(11)$ \\
$B-V$ & $0.51(2)$ & $0.82(2)$ & $0.33(1)$ \\
\hline$i_{\mathrm{A}}(\operatorname{deg})$ & $64.0(1.0)$ & & \\
$a_{\mathrm{A}}\left(R_{\odot}\right)$ & $7.2(4)$ & & \\
$V, B-V$ total & $11.30(11), 0.48(2)$ & \\
\hline
\end{tabular}

nitude of each star Aa and $\mathrm{Ab}$ in the eclipsing binary from its temperature, radius, and surface gravity. The $V$ magnitude of the blue straggler B then follows from the fitted luminosity scaling. The second constraint is that the implied mass of the blue straggler is roughly consistent with its place in the colour-magnitude diagram. That is, the radius of the blue straggler $\mathrm{B}$ can be computed from the distance, the $V$ magnitude, and the temperature. Since the gravity of the blue straggler B is specified in the models, its mass can then be computed. The blue colour of S 1082 requires a relatively hot third light $\operatorname{star}(\approx 7500 \mathrm{~K})$, and its surface gravity must be near $\log g=4.25$ in order for the mass to be near $\approx 1.7 M_{\odot}$. The derived astrophysical parameters for the adopted model are summarised in Table 5 . The errors on the parameters were estimated from the $\chi^{2}$ values generated in the various grid searches. These error estimates may be too small, given the complicated nature of the model. The errors on the masses were taken to be on the order of $15 \%$, as judged from the quality of the sinusoid fits. The light curves of the three components are shown in Fig. 8, and a cartoon of the binary at three phases in Fig. 9.

We have assumed that the stars are not spotted and that the eclipsing binary has a circular orbit. A violation of either one of these assumptions could alter the light curves to produce the small systematic deviations seen in the residuals. Bright or dark spots could either add or remove light at certain phases, complicating the analysis. Given the rather large number of free parameters we have now we did not consider adding spots at this time. If this binary is part of a triple, then the orbit could be eccentric (see Sect. 5). A slight eccentricity ( $e \approx 0.05$ say) could cause the maxima to be asymmetric and the minima to be shifted slightly in phase. Our current velocity curves do not have enough phase coverage to place meaningful constraints on the eccentricity, so any firm conclusions on the eccentricity will have to await the arrival of additional data.

Figure 10 shows the decomposition of S 1082 into its components in a colour-magnitude diagram of M67. Star 


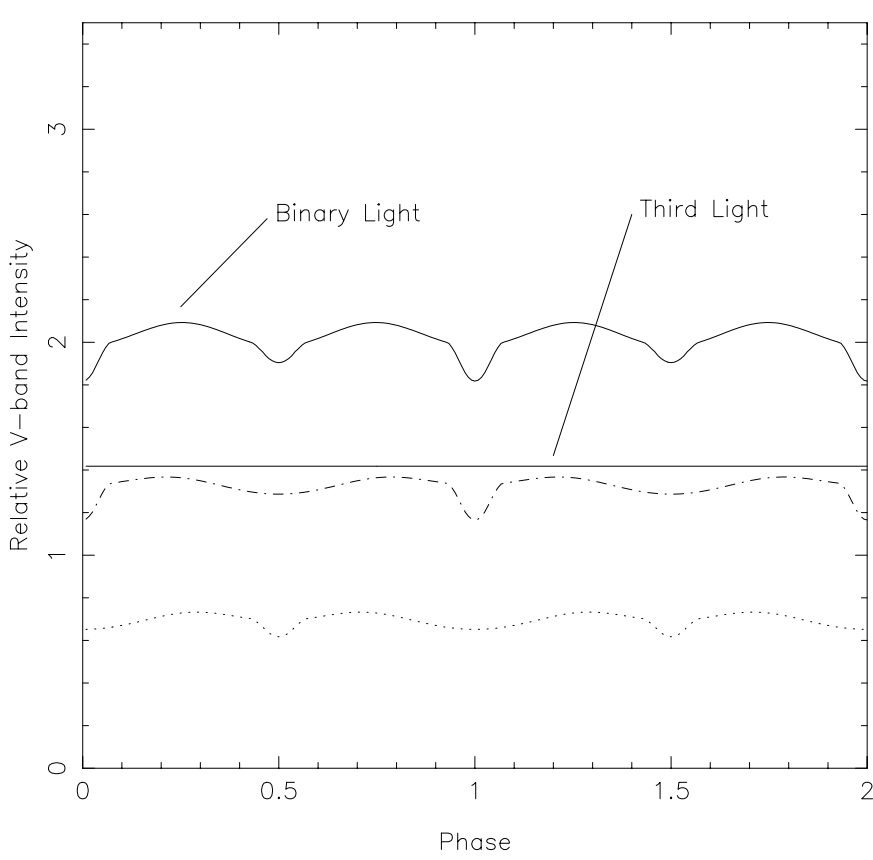

Fig. 8. Light curves of the three components in S 1082 (dasheddotted line indicates the primary Aa in the eclipsing binary).

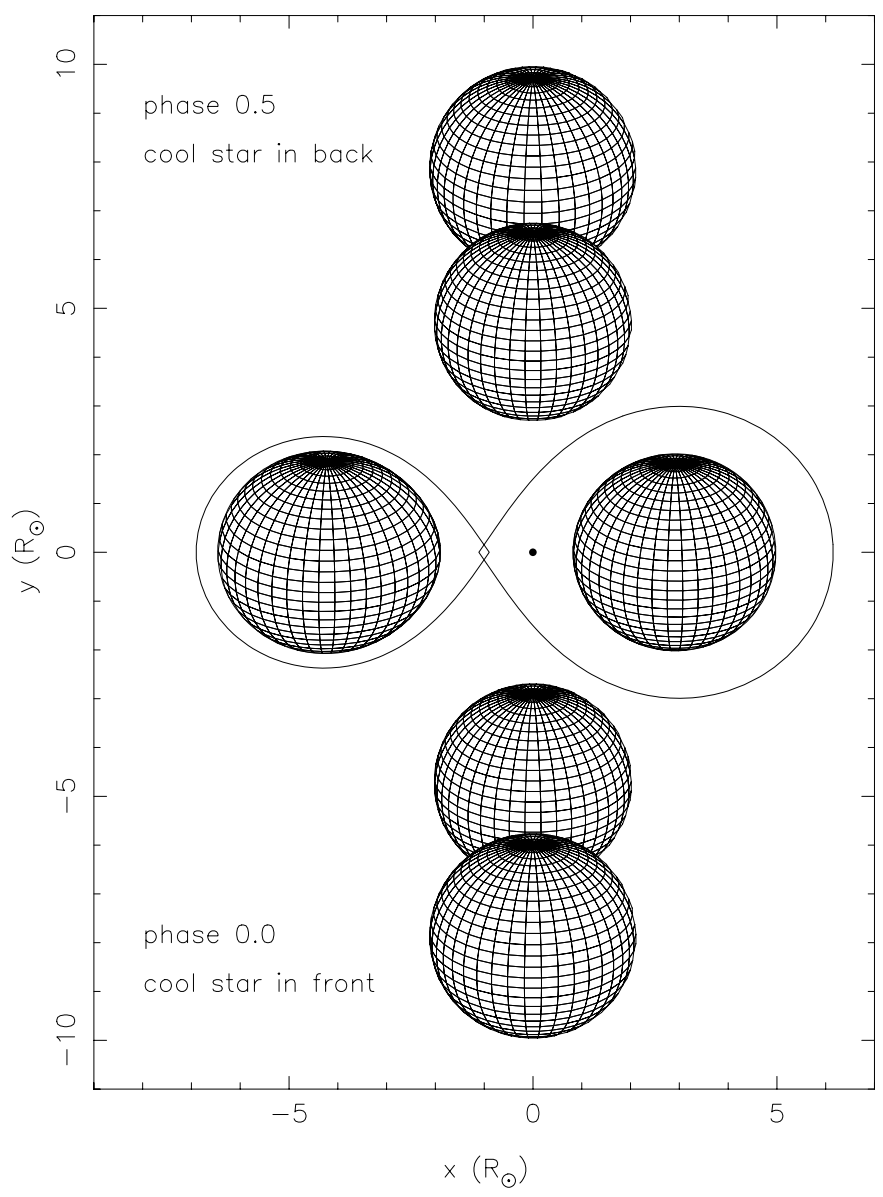

Fig. 9. Cartoon of the configuration at three orbital phases of the inner binary according to the model listed in Table 5. Note that the Roche-lobes of the two stars Aa and Ab slightly overlap due to the fact that neither star corotates with the orbit.
Table 6. Times of primary minimum. From left to right: observed time of minimum; $\mathrm{O}-\mathrm{C}$ difference (in days) between the observed and computed time of eclipse; cycle number of the eclipse with respect to the $T_{0}$ of Table 4 . The errors in the observed times of minimum and the $\mathrm{O}-\mathrm{C}$ values are 0.005 days.

\begin{tabular}{rrrl}
\hline HJD & O-C & cycle & source \\
-2440000 & & & \\
\hline 3191.036 & -0.010 & -1360 & Simoda $(1991)^{a}$ \\
4643.250 & 0.000 & 0 & Goranskij et al. (1992) \\
5325.586 & 0.013 & 639 & idem \\
6773.492 & -0.013 & 1995 & idem \\
7861.605 & 0.014 & 3014 & idem \\
7920.333 & 0.014 & 3069 & idem \\
7944.869 & -0.010 & 3092 & idem ${ }^{b}$ \\
11218.744 & -0.001 & 6158 & run 1 \\
11539.078 & -0.006 & 6458 & run 2 \\
\hline
\end{tabular}

${ }^{a}$ Simoda observed two consecutive primary eclipses. These data were combined to measure one time of minimum.

${ }^{b}$ Based on a measurement of a secondary eclipse which we convert to a primary eclipse by adding half a period. We note that if the orbit of the eclipsing binary is eccentric, the two eclipses need not be separated by half a period.

$\mathrm{Ab}$ is located near the $4 \mathrm{Gyr}$ isochrone that provides the best fit to the observed main-sequence, subgiant and giant stars (Pols et al. 1998). Hence the position of star Ab is consistent with the expected value based on its mass. The positions of star Aa and the blue straggler B are a bit more uncertain since one can "trade off" flux between the two stars (i.e. the blue straggler B can be made brighter at the expense of star Aa in the binary). We note that the star $\mathrm{Ab}$ is always located (within the errors) on the $4 \mathrm{Gyr}$ isochrone. The error bars shown on the $V$ magnitudes reflect the uncertainties for our adopted model which produces an overall $V$ magnitude of all three stars close to the observed value. In any event, the hotter star Aa is subluminous by at least 2 mag in $V$ (compare with the track for a $2.2 M_{\odot}$ star). The position of the blue straggler lies to the red of the extension of the main-sequence as defined by the $1 \mathrm{Myr}$ isochrone.

In order to obtain observed times of primary minimum we use the model light curve as a template to fit the data near the primary minima observed during run 1 and run 2. Seven additional times of minimum are obtained from fitting the data in Table 3 of Goranskij et al. and from the data points of Simoda (1991) (see first column of Table 6). A straight line is fitted to the observed times of minimum to find a new period and $T_{0}$ (see Table 4). The period thus derived is compatible with the period listed by Goranskij et al. (Eq. (1)). We use the new ephemeris to compute observed minus computed $(\mathrm{O}-\mathrm{C})$ times of primary eclipse and the corresponding cycle number with respect to $T_{0}$ (second and third column of Table 6 ). The peak-to-peak amplitude $\Delta \mathrm{O}-\mathrm{C}$ is $\sim 39 \mathrm{~min}$. If these variations are real and caused by the motion of the eclipsing 


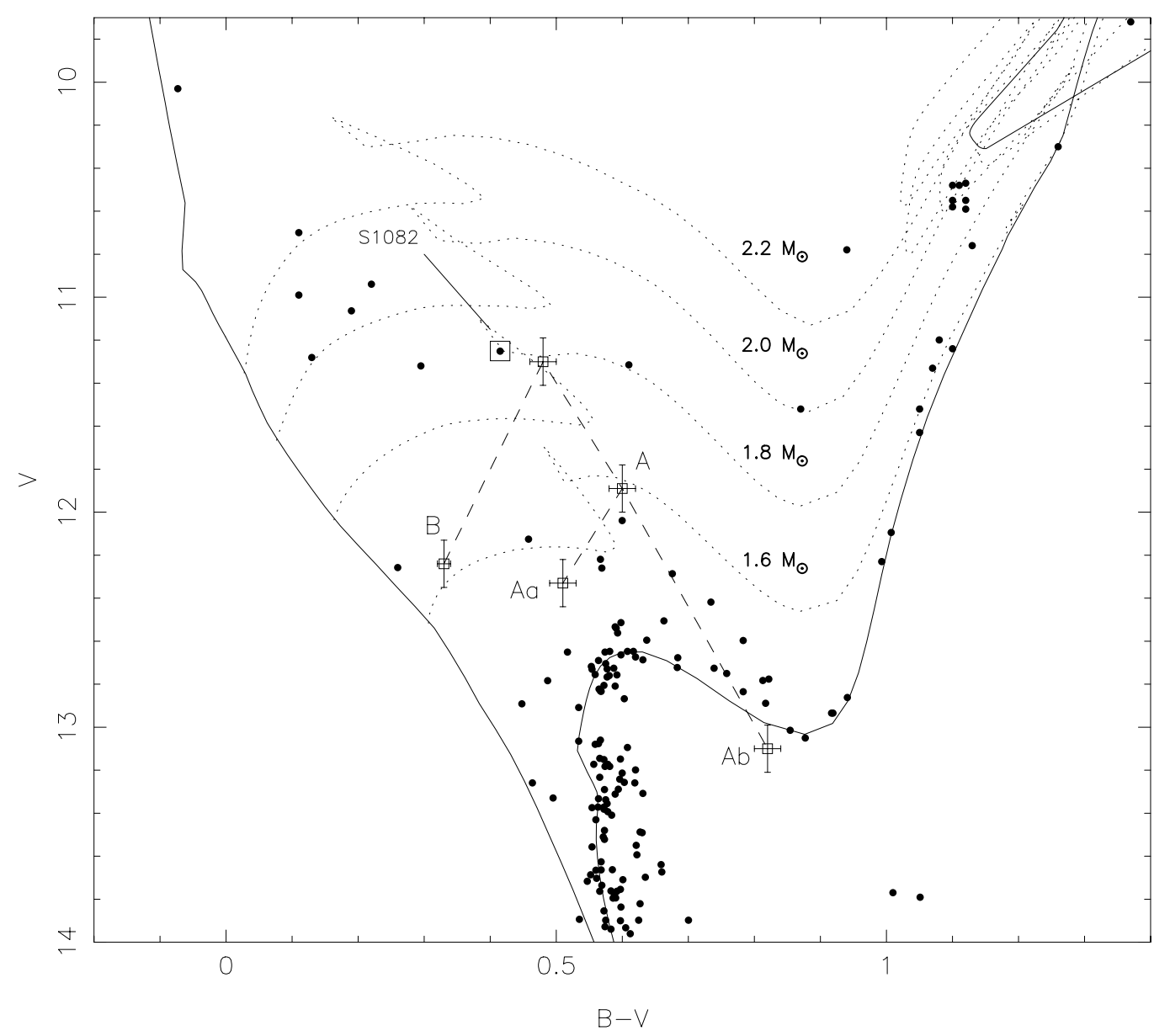

Fig. 10. Colour-magnitude diagram of M 67 that shows the decomposition of S 1082 into a blue straggler (B) and stars Aa and $\mathrm{Ab}$ in the eclipsing binary (A); a dashed line connects their positions. The observed location of S 1082 is indicated with a box; the height of the square equals the depth of the primary eclipse. The dotted lines are evolutionary tracks for 1.6, 1.8, 2.0 and $2.2 M_{\odot}$ stars corrected for the distance modulus and reddening of M67 ( $Z=0.02$, Pols et al. 1998). The 4 Gyr-isochrone is included to indicate the expected positions of cluster members; the 1 Myr isochrone is included to give an estimate for the location of the ZAMS (Pols et al. 1998). $B$ and $V$ magnitudes of M 67 stars are from Montgomery et al. (1993). Only stars with a proper-motion membership probability $>0.8$ (Girard et al. 1989) are plotted.

binary around a third body it would correspond to a minimum semi-major axis in the outer (o) orbit of the binary (b) $a_{\mathrm{o}, \mathrm{b}} \sin i_{\mathrm{o}}=1 / 2 c \Delta \mathrm{O}-\mathrm{C}=2.3 \mathrm{AU}$. Assuming that the blue straggler (B) has a mass compatible with its position in the colour-magnitude diagram (about $1.7 M_{\odot}$ ) this corresponds to a minimum value of the semi-major axis of the total system $a_{\mathrm{o}} \sin i_{\mathrm{o}}=a_{\mathrm{o}, \mathrm{b}} \sin i_{\mathrm{o}}\left(1+M_{\mathrm{A}} / M_{\mathrm{B}}\right) \approx 8.4 \mathrm{AU}$; combined with the total mass of the system of $6.1 M_{\odot}$ and Kepler's third law this gives a minimum period of 10 years. This is not compatible with the period of $\sim 3$ years found by Milone (1991). We conclude that not all the variation in $\mathrm{O}-\mathrm{C}$ is due to light-travel time in the outer orbit. There is no evidence for periodicity in the $\mathrm{O}-\mathrm{C}$ times, although the time baseline is somewhat short and the coverage is somewhat spotty.

\section{Discussion}

Our new observations of S 1082 confirm the eclipses reported by Goranskij et al. (1992), the small radial-velocity variations of the narrow lines in the spectrum (Mathieu et al. 1986) and the time-variation of a broad-lined component seen in high-resolution spectra (van den Berg et al. 1999; Shetrone \& Sandquist 2000). In addition, we now have found that the radial-velocity shifts of the broadlined component and of a third component in the spectra vary on the photometric period. This clearly demonstrates that the narrow-lined star, which is a blue straggler on its own, is not part of the eclipsing binary - this solves the seeming contradiction in the properties of this system. The broad variable component of the $\mathrm{H} \alpha$ line discussed by van den Berg et al. (1999) is the Stark-broadened $\mathrm{H} \alpha$ line of the hot component Aa of the inner binary. The ultraviolet flux measured by Landsman et al. (1998), higher than expected for a star at the $B-V$ colour of $\mathrm{S} 1082$, is only partly explained as a consequence of the bluer colour, i.e. higher temperature of the third star.

Comparison with Figs. 2 and 3 of Dempsey et al. (1993) shows that the X-ray luminosity of S 1082 (Belloni et al. 1998) is typical for a subgiant in an RS CVn system. 


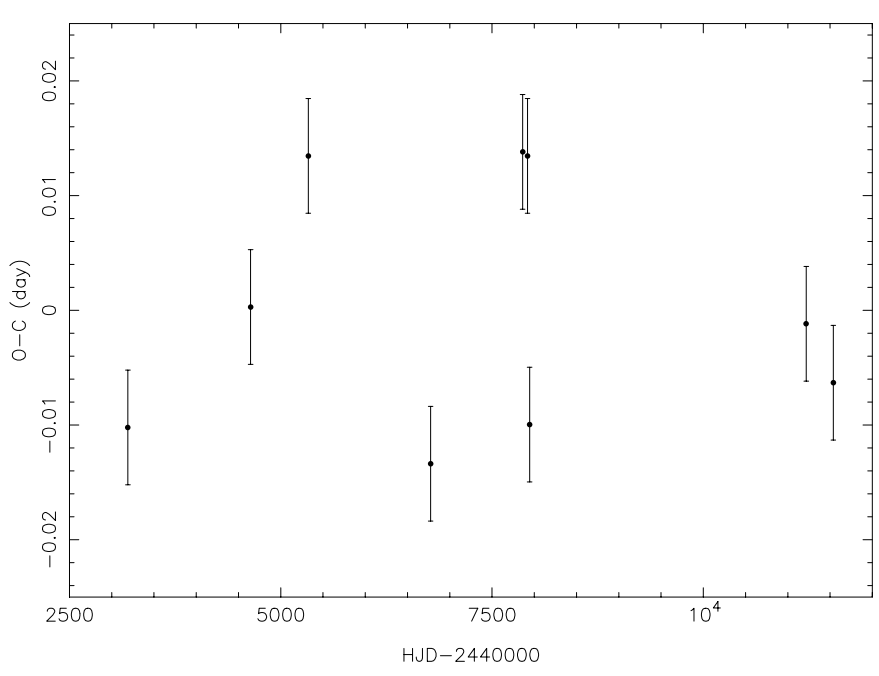

Fig. 11. O-C times of the primary eclipse in days versus heliocentric Julian date $(-2440000)$ of the measurement.

The X-ray emission could therefore be caused by magnetic activity in the rapidly rotating subgiant Ab. Thus, our model can explain the X-rays of S 1082.

Our solution to the photometry is symmetric (see Fig. 8) and does not explain the asymmetry between phases 0.25 and 0.75 ; nor the variability in the form of the secondary eclipse between our first two observation runs. This indicates that variable spots are present and accordingly that the best-fit parameters of the system are subject to some additional uncertainty. However, we do not think our main conclusions are affected.

We think that the eclipsing binary forms a bound triple with the third star, for two reasons. First, the radialvelocity measurements of the third star B - the narrowlined system in S 1082 - indicate that it is in a $\sim 1000$ day orbit around a companion. The systemic velocities of both the 1000 day and the 1.07 day binaries are compatible with the radial velocity of the cluster. A chance alignment of two such bright cluster members is unlikely. Second, if we relax the constraint that the binary is at the cluster distance in solving the photometry, we find that the best solutions tend to be an Algol binary at higher mass $\left(\gtrsim 4 M_{\odot}\right)$ and larger distance (about twice the cluster distance). A high-mass binary at such a large distance from the galactic disk is unlikely.

Assuming then that the binary forms a hierarchical triple with the third star, in M67, we note that the 1- $\sigma$ lower bound to the mass of the binary is at about three times the turnoff mass of the cluster. The formation of the binary must thus have involved at least three stars. The third star is a blue straggler on its own account, and thus according to most current models its formation involves two stars. We have to conclude that the formation of $\mathrm{S} 1082$ required the interaction of no less than five stars!

This interaction may have started with a binary-binary encounter, in which two stars collided directly and merged; one of the remaining two stars ended in a close orbit around the merger, the fourth star in a wide orbit around the inner binary. The fourth star must be a blue straggler, which either was already present in the original encounter or was later exchanged into the system. That triples are formed easily by binary-binary encounters, and that they live long enough to undergo subsequent exchange encounters with a binary, is shown for example by the computations of Aarseth \& Mardling (2001). It is less obvious that mergers are common in such encounters, as most binary-binary encounters are between relatively wide systems. Merger products tend to be subluminous (Sills et al. 2001), in accordance with the properties of the more massive star Aa in the inner binary. The main problem with this scenario for the formation of the S 1082 system is that a merger product is only subluminous for a very limited period; comparable to its thermal time scale. This reduces the available time within which the outer star of the first encounter is exchanged with a binary or blue straggler in a subsequent encounter, and indicates that the blue straggler was present in the initial binary-binary encounter. Alternatively, an initial encounter between two triple systems can have led immediately to the currently observed configuration. In any case, the probability of catching the merger product while it is strongly subluminous is disconcertingly small.

Aarseth \& Mardling (2001) note that the inclination of the outer orbit in a hierarchical triple with respect to the inner orbit can induce a large eccentricity in the inner orbit; subsequently, tidal forces in the inner orbit cause it to shrink. Thus, the inner orbit may be eccentric, and smaller than in the past. Our solution for the inner binary implies that the cool star has a radius which is a sizeable fraction of the binary separation $R_{\mathrm{Ab}} / a_{\mathrm{A}} \approx 0.3$. According to Eq. (2) of Verbunt \& Phinney (1995) the circularisation time scale of the inner binary $\mathrm{A}$ is $\sim 10^{3}$ year; the eccentricity of $\mathrm{A}$ and asynchronicity of $\mathrm{Ab}$ thus will be determined by the competing effects of the perturbation by the outer star B and the tidal forces in the inner binary. Only if the latter are substantially reduced with respect to the Zahn (1977) formulation - as suggested by e.g. Goodman \& Oh (1997) - do we expect measurable eccentricity and asynchronicity.

Whereas our new observations have allowed us to resolve the apparent contradictions in the earlier data and to determine the system parameters, we conclude that these parameters - in particular those of the primary Aa of the inner binary - are hard to understand in terms of standard stellar and binary evolution, even when stellar encounters and mergers are taken into account. This situation is remarkably similar to that in the study of two other members of M67, located below the subgiant branch in the colour-magnitude diagram, both of which turn out to be binaries (Mathieu et al. 2001, in preparation); and indeed to our lack of understanding of the blue straggler population in the cluster. Many of these stars may be mergers, which raises the question whether the object that results when two stars merge may follow an evolutionary track which is very different from that of an ordinary star of the 
same mass; and whether they can do so for a period of time which significantly exceeds the thermal time scale.

Further studies of the remarkable triple system S 1082 should provide a better-sampled radial-velocity curve, necessary to determine whether the inner binary is eccentric, and to measure its systemic velocity more accurately to establish cluster membership. Better-quality light curves may also serve to improve the accuracy of the inner orbit, e.g. if the inner orbit is eccentric the separation of the eclipses is different of 0.5. A long-term sampling of the light curves will be required to improve the interpretation of the $\mathrm{O}-\mathrm{C}$ in the timings of the primary eclipse.

Acknowledgements. The authors wish to thank M. Janson, R. Dijkstra, G. Geertsema, R. Cornelisse and G. Nelemans for doing part of the observations. We also want to thank D. Latham for discussions. M.vdB. is supported by the Netherlands Organization for Scientific Research (NWO).

\section{References}

Aarseth, S., \& Mardling, R. 2001, in Evolution of Binary and Multiple Star Systems, ed. P. Podsiadlowski, et al., ASP Conf. Ser., 229, in press [astro-ph/0011514]

Bailyn, C. D. 1995, ARA\&A, 33, 133

Belloni, T., Verbunt, F., \& Mathieu, R. D. 1998, A\&A, 339, 431

Bevington, P. R. 1969, Data reduction and error analysis for the physical sciences (New York: McGraw-Hill)

Dempsey, R. C., Linsky, J. L., Fleming, T. A., \& Schmitt, J. H. M. M. 1993, ApJS, 86, 599

Girard, T. M., Grundy, W. M., López, C. E., \& van Altena, W. F. 1989, AJ, 98, 227

Goodman, J., \& Oh, S. P. 1997, ApJ, 486, 403

Goranskij, V. P., Kusakin, A. V., Mironov, A. V., Moshkaljov, V. G., \& Pastukhova, E. N. 1992, Astron. Astrophys. Trans., 2, 201

Gray, D. F. 1992, The observation and analysis of stellar photospheres (Cambridge University Press)
Gray, R. O., \& Garrison, R. F. 1989, ApJS, 69, 301

Hauschildt, P. H., Allard, F., \& Baron, E. 1999a, ApJ, 512, 377

Hauschildt, P. H., Allard, F., Ferguson, J., Baron, E., \& Alexander, D. R. 1999b, ApJ, 525, 871

Honeycutt, R. K. 1992, PASP, 104, 435

Hurley, J. R., Tout, C. A., Aarseth, S. J., \& Pols, O. R. 2001, MNRAS, 323, 630

Kurucz, R. L. 1979, ApJS, 40, 1

Landsman, W., Bohlin, R. C., Neff, S. G., et al. 1998, AJ, 116, 789

Latham, D. W., \& Milone, A. A. E. 1996, in The origins, evolution, and destinies of binary stars in clusters, ed. E. Milone, \& J.-C. Mermilliod, ASP Conf. Ser., 90, 385

Leonard, P. J. T. 1996, AJ, 470, 521

Mathieu, R. D., Latham, D. W., Griffin, R. F., \& Gunn, J. E. 1986, AJ, 92, 1100

Mathys, G. 1991, A\&A, 245, 467

Milone, A. A. E. 1991, Ph.D. Thesis, Univ. of Córdoba

Milone, A. A. E., \& Latham, D. W. 1992, in Evolutionary Processes in interacting binary stars, ed. Y. Kondo, et al., 475

Montgomery, K. A., Marshall, L. A., \& Janes, K. A. 1993, AJ, 106,181

Nissen, P. E., Twarog, B. A., \& Crawford, D. L. 1987, AJ, 93, 634

Orosz, J. A., \& Hauschildt, P. H. 2000, A\&A, 364, 265

Pols, O. R., Schröder, K.-P., Hurley, J. R., Tout, C. A., \& Eggleton, P. P. 1998, MNRAS, 298, 525

Shetrone, M. D., \& Sandquist, E. L. 2000, AJ, 120, 1913

Sills, A., Faber, J. A., Lombardi Jr, J. C., Rasio, F. A., \& Warren, A. R. 2001, ApJ, 548, 323

Simoda, M. 1991, IBVS 3675

Stryker, L. L. 1993, PASP, 105, 1081

van den Berg, M., Stassun, K., Verbunt, F., \& Mathieu, R. D. 2001, A\&A, submitted

van den Berg, M., Verbunt, F., \& Mathieu, R. D. 1999, A\&A, 347,866

Verbunt, F., \& Phinney, E. S. 1995, A\&A, 296, 709

Wilson, R. E. 1990, ApJ, 356, 613

Zahn, J.-P. 1977, A\&A, 57, 383 